\section{Prämiertes Welttheater}

Prof. Dr. Dr. Johannes Ring, Direktor der Klinik und Poliklinik für Dermatologie und Allergologie am Biederstein der Technischen Universität München wurde mit dem „Health Media Award 2010" ausgezeichnet. Der Preis wird durch die Stiftung für Gesundheit in Hamburg in Zusammenarbeit mit dem Medienpartner EEC Network für hervorragende Leistungen im Bereich der Gesundheitskommunikation verliehen. Ausgezeichnet wurde das von Ring verfasste und von Mitarbeitern beim World Allergy Congress in München uraufgeführte allergologische Musical „L' Allergica Comedia“, das auf den Spuren von Dante Alighieri die Probleme sowohl der Allergieforschung als auch der -behandlung liebevoll humoristisch illustriert.

\section{Neue Online-Publikation}

"Clinical and Translational Allergy" (CTA) heißt eine neue Open-access-onlinePublikation, die von der European Academy of Allergy and Clinical Immunology (EAACI) herausgegeben wird. Die Zeitschrift soll in erster Linie wissenschaftliche Arbeiten und Übersichten zur Grundlagen- und klinischen Forschung auf dem Gebiet der Allergologie publizieren. Die Begutachtung soll zügig vonstattengehen. Um die freie Bereitstellung im Internet zu finanzieren, sollen die Autoren für die Publikation eine Nominalgebühr entrichten. Mehr dazu ist online unter www.ctajournal.de nachzulesen.

\section{Vorzeigbarer Aktionsplan}

Wenn sich das Gesundheitsministerium nicht um die Allergie kümmert, dann muss es eben das Bundesministerium für Ernährung, Landwirtschaft und Verbraucherschutz machen, dachte sich Horst Seehofer 2007 und rief den „Aktionsplan gegen Allergien“ ins Leben. Seine Nachfolgerin Ilse Aigner hat das Projekt fortgeführt. Die zugehörige Website www.aktionsplanallergien.de hat sich inzwischen zu einem respektablen Portal gemausert, das umfassend zum Thema Allergien informiert. Betroffene und Interessierte erhalten eine Orientierungshilfe und praktische Tipps zu verschiedenen Lebensbereichen. Ergänzend finden sie dort Links zu weiterführenden Informationen. Welche Projekte der Aktionsplan aktuell ansonsten verfolgt, lässt sich ebenfalls online nachlesen.

\section{Preisfrage: Was passiert, wenn die Lunge umbaut?}

\author{
Die Symptomatik des Asthma bronchiale wird durch Umbau- \\ prozesse in den Atemwegen mitbestimmt. Welche Rolle die \\ epitheliale-mesenchymale Transition dabei spielt, interessierte die \\ Kanadierin Dr. Tillie-Louise Hackett. Im April wurde ihre Neugier \\ mit dem „International Klosterfrau Research Award of Airway \\ Diseases in Childhood 2011" belohnt.
}

ei Asthma bronchiale kommt es zu einem chronischen Remodeling der Atemwege. Das bedeutet strukturelle Veränderungen der Lungenwände, unter anderem mit Hypertrophie des glatten Muskelgewebes, Membranverdickung, Angiogenese sowie submukosaler Fibrose. Dieser Umbauprozess trägt wesentlich zur kontinuierlichen Abnahme der Lungenfunktion und damit auch zur Symptomatik der Erkrankung bei. Seine Mechanismen sind aber noch unzureichend verstanden.

In den Fokus des wissenschaftlichen Interesses ist in diesem Zusammenhang seit einiger Zeit die epitheliale-mesen- chymale Transition (EMT) gerückt. Sie könnte im Lungengewebe für einen erhöhten Pool an Fibroblasten sorgen, welche die Synthese und Sekretion extrazellulärer Matrixproteine induzieren. Dr. Tillie-Louise Hackett von der University of British Columbia in Vancouver, Kanada, konnte zeigen, dass sich mit dem Transformationswachstumsfak tor(TGF)- $\beta 1$ in humanen Lungenepithelzellen eine EMT induzieren lässt und dass dieser Prozess bei Gesunden und Asthmakranken unterschiedlich verläuft. Für ihre Arbeiten erhielt sie anlässlich der Jahresversammlung der Gesellschaft für Pädiatrische Immunologie im April

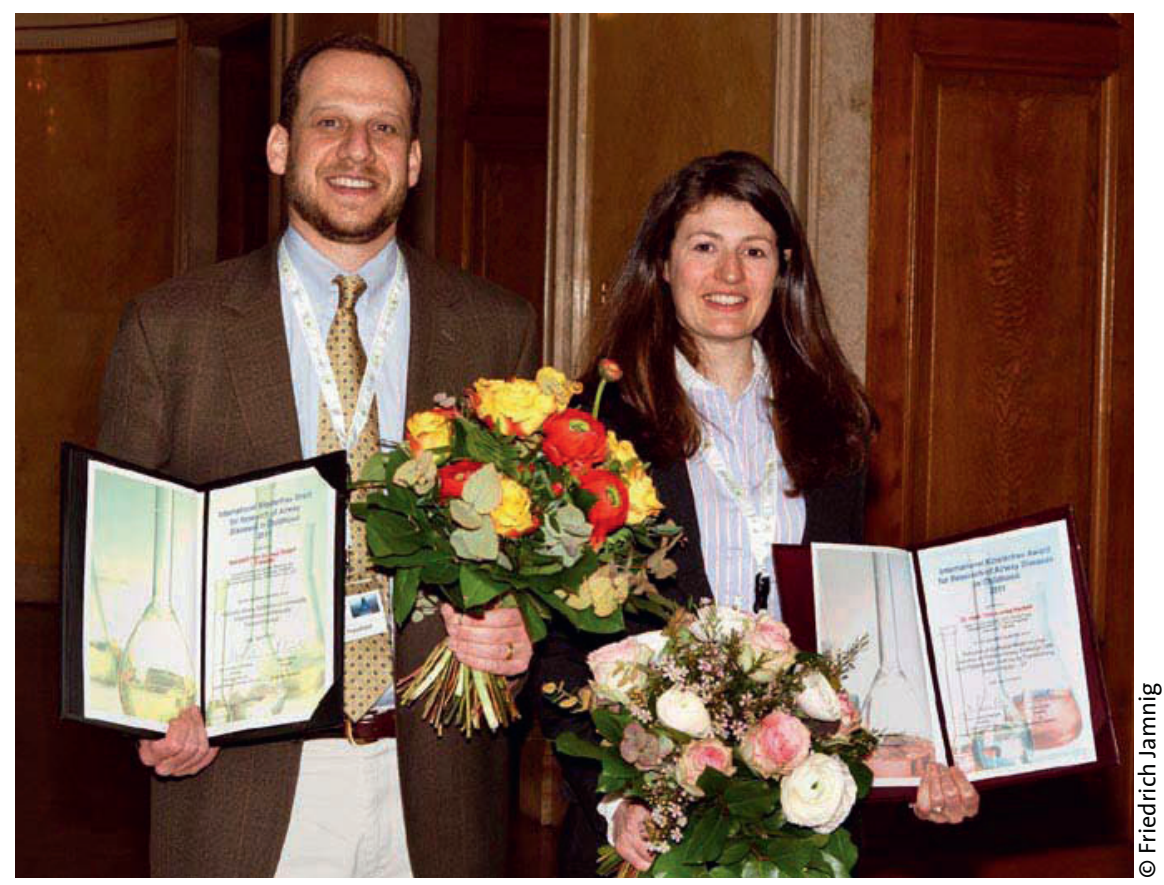

Dr. Robert J. Freishtat und Dr. Tillie-Louise Hackett wurden für ihre Beiträge zum Verständnis der Grundlagen des Atemwegsremodeling bei Asthma mit dem ,International Klosterfrau Grant for Research of Airway Diseases in Childhood“ bzw. dem „International Klosterfrau Research Award of Airway Diseases in Childhood 2011" ausgezeichnet. 